\title{
COMMUNICATION
}

Cite this: DOI: $10.1039 / \mathrm{x} 0 x \times 00000 x$

\section{Electrohydrodynamic removal of non-specific colloidal adsorption at electrode interfaces †}

\author{
Sakandar Rauf, ${ }^{a}$ Muhammad J. A. Shiddiky*a and Matt Trau*a,b
}

Received 00th January 2013,

Accepted 00th January 2013

DOI: $10.1039 / \times 0 \times x 00000 x$

www.rsc.org/

This communication reports the use of an electrohydrodynamic surface shear force to selectively manipulate colloid-surface interactions. We demonstrate the selection of strongly (specifically) bound biomolecule-functionalized colloidal beads over more weakly (non-specifically) bound beads using a tuneable alternating current electrohydrodynamic (ac-EHD) force, which drives lateral fluid motion within a few nanometers of an electrode surface. By externally "tuning" the strength of the ac-EHD force, we demonstrate a significant enhancement of capture efficiency for specifically bound colloids, alongside a removal of the adsorption of non-specific colloidal beads-a process which may be observed in real-time.

Colloidal micro and nanoparticles are ubiquitous owing to their diverse properties and numerous applications. ${ }^{1}$ Over the past few decades, biologically functionalized colloidal particles have received wide attention as transduction labels in bioassays for the detection of molecular and cellular targets such as DNA, ${ }^{2}$ proteins ${ }^{3}$ or cancer cells. ${ }^{4}$ Often, these assays are performed on solid surfaces that are functionalized with different affinity reagents (e.g., antibody biomolecules) and involve readouts such as fluorescence, ${ }^{3}$ surface enhanced Raman scattering ${ }^{5}$ or electrochemistry. ${ }^{6}$ Colloidal particles, however, tend to adsorb non-specifically to solid surfaces, e.g., on gold electrode surfaces, and thus can significantly affect the specificity and sensitivity of solid-phase biomolecular assays. Generically, the problem of selectively capturing colloids with specific surface chemistry versus colloids which bind to solid surfaces due only to non-specific physical forces (such as electrostatic or van der Waals colloidal forces) is also potentially of broad interest to the chemistry community.

${ }^{a}$ Australian Institute for Bioengineering and Nanotechnology (AIBN), The University of Queensland, Corner of College and Coopers Roads, Brisbane, QLD 4072, Australia. ${ }^{b}$ School of Chemistry and Molecular Biosciences, University of Queensland, Brisbane, QLD 4072, Australia E-mail: m.shiddiky@uq.edu.au and m.trau@uq.edu.au: †Electronic Supporting Information (ESI): Experimental and supporting data, see DOI: $10.1039 / c 000000 x /$
Currently, several methods have been reported to reduce nonspecific adsorption of colloidal particles or molecules., ${ }^{7}$ While all these methods demonstrate low non-specific adsorption, their practical application is restricted due to the longer analysis time, complicated chemistries, and multiple washing steps.

Herein, we report the utilization of an alternating current electrohydrodynamic (ac-EHD) induced physical force that can physically displace non-specifically bound colloidal particles and simultaneously enhance the capture of specific colloids on the surface of a modified electrode. To demonstrate the utility of this electrohydrodynamic phenomenon, we present data on a device containing an asymmetric pair of electrodes to specifically select streptavidin-modified fluorescent beads in presence of the carboxylmodified non-specific fluorescent beads. The use of ac-EHD to manipulate colloidal objects on the surface of flat electrodes was originally demonstrated and modelled by Trau et al., ${ }^{9}$ where fluid flow arises from electrical body forces acting on free charges generated in solution. By tuning the microscopic EHD fluid flow, Trau et al. demonstrated that designer colloidal structures can be assembled or sheared away. More recently, fluid flow engendered from asymmetric electrodes have been employed to assemble and pattern colloids into linear chains and other clustered ordered structures. ${ }^{10,11}$ To date, however, there is no report that uses ac-EHD forces to physically displace non-specific colloids from solid surfaces whilst simultaneously enhancing capture efficiency.

As can be seen in Fig. 1, large (ring type electrode) and small (inner circular electrode) electrodes in an asymmetric pair of the microelectrode form the cathode and anode (or vice versa) of an electrolytic cell. Upon the application of an alternating potential difference across these microelectrodes, a non-uniform field $(E)$ induces charges within the double layer of each electrode. These induced charges experience a force $F\left(F=\rho E_{\mathrm{t}}\right.$, where $\rho=$ charge density, $E_{\mathrm{t}}=$ tangential component of $E$ ) with the asymmetric geometry giving rise to lateral variation in the total amount of double layer charges and spatial distribution of charges on the electrode 

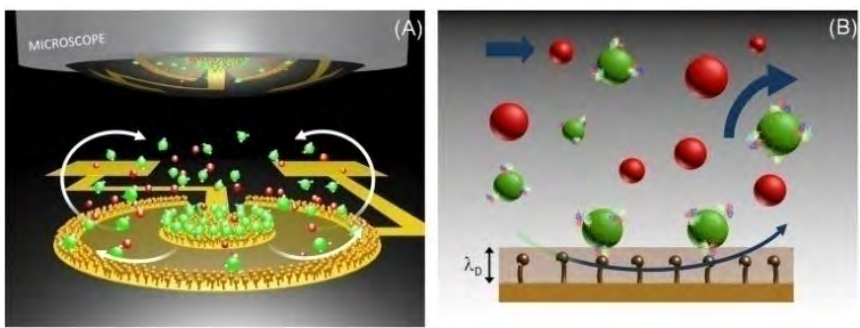

Fig. 1 (A) Schematic representation of the approach adopted for real-time visualization of the electrohydrodynamic force to enhance the capture efficiency of the colloids by increasing the number of sensor (functionalized surface)-target collisions, which is the result of the improved colloidal transport under ac-EHD fluid flow. (B) Schematic representation of capture of specific and removal of non-specific colloids via tuning the surface shear forces within the electrical double layer of the surface.

surface. $^{12}$ Consequently, the resultant non-uniform force due to induced charges on the larger electrode $\left(F_{\mathrm{L}}\right)$ is stronger than on the smaller $\left(F_{\mathrm{s}}\right)$ electrode (i.e., $F_{\mathrm{L}}>F_{\mathrm{s}}$ ), resulting in a lateral flow towards the large electrode. The steady flow towards the large electrode causes any beads in solution to be dragged by the flow. One of the attractive feature of this phenomenon is that the induced charges strictly remain within the double layer of the electrodes $\left(\lambda_{D}=1 / \mathrm{K}=\right.$ double layer thickness ${ }^{1}$ ). In our case (for $1 \mathrm{mM}$ phosphate buffer saline at $25^{\circ} \mathrm{C}$ ), the value of $\lambda_{D}$ was calculated to be $10-12 \mathrm{~nm}$ using the equation described previously. ${ }^{1}$ This means that these shear forces engender fluid flow vortices and create fluid mixing that can displace weakly bound non-specific colloidal particles by tuning fluid shear forces at the nanometre distances from the electrode surface. To investigate this phenomenon, devices with asymmetric electrode pairs were fabricated maintaining edge-to-edge distances between inner and outer electrode $\left(\mathrm{d}_{\mathrm{e}-\mathrm{e}}\right)=50,200$ and $1000 \mu \mathrm{m}$ (ESIt and Fig. S1 for details). The diameter of the inner electrode and the width of the outer ring electrode were 250 and $30 \mu \mathrm{m}$ respectively for all the devices (ESIt and Fig. S1 for details). A $0.4 \mathrm{~mm}$ thick PDMS spacer with a $5 \mathrm{~mm}$ diameter hole was used to create the analysis chamber which was then filled with $15 \mu \mathrm{L}$ mixture solution containing designated amounts of specific and non-specific beads, and finally covered with a glass cover slip to complete the device.

To visualize the ac-EHD surface shear effect in real-time, the surface of the gold electrodes was first modified with biotin using cysteamine hydrochloride and sulfo-NHS-LC-biotin linker (ESIt). Streptavidin modified (Dragon green dye) and carboxyl modified (Sun coast yellow appears red colour) fluorescent beads were used as "specific" and "non-specific" targets, respectively. A dual band pass filter set was used to image both the beads simultaneously (ESIt). Fig. $2 \mathrm{~A}$ shows the brightfield image of the device containing an asymmetric pair of electrodes and fluorescence micrographs upon the capture of streptavidin labelled beads in presence of the nonspecific carboxyl beads under the ac-EHD field strength of frequency $(f) 100 \mathrm{~Hz}$ and amplitude $\left(V_{\mathrm{pp}}\right) 4 \mathrm{~V}$ (inner electrode diameter $250 \mu \mathrm{m}$ and $\mathrm{d}_{\mathrm{e}-\mathrm{e}}=1000 \mu \mathrm{m}$ ). Fig. 2B shows the snapshots of real-time capture of specific beads and displacement of non-specific beads (Fig. S2, Video1, ESIt). The arrows in Fig. 2B are pointing the green and red coloured beads indicating the position of the beads on the electrode surface over the 4s-period of electrohydrodynamic induced capture process. These data clearly indicate that surface shear forces created

by the ac field were strong enough to displace the non-specific carboxyl beads (red) while simultaneously enabled the enhanced capture of specific streptavidin beads (green) on the biotin-modified surface.

A control experiment was performed to check the capture performance of the devices without the use of ac-EHD field (Fig. S3, ESIt). A significantly low number of green and red beads were attached to the electrode surface after 24 hour incubation of devices in the mixture solution (i.e., $50 \%$ specific $+50 \%$ non-specific). However, under the ac-EHD condition, the number of the captured specific beads increased significantly due to the enhanced strepatvidin-biotin collisions. In another control experiment, we checked the capture performance of our ac-EHD method using a device with bare gold electrode (Fig. S4, ESIt). As expected a relatively low number of specific and non-specific beads adsorbed onto the unmodified surface in comparison to the biotinfunctionalized surface. Notably, the number of the specific beads at biotin-functionalized surface were significantly increased while the non-specific beads were almost similar in both cases. These data indicate that the surface shear forces generated upon the application of an ac field can (i) selectively enhance the capture of strepatvidin beads on biotin functionalized surface and (ii) effectively displace the non-specific species or beads from the electrode surface.

The effect of different field strengths on the specific capture of streptavidin beads was evaluated by changing the frequency and amplitude of the applied ac field. It was found that at the field strength of $f=100 \mathrm{~Hz}$ and $V_{\mathrm{pp}}=4 \mathrm{~V}$, the shear forces were strong enough to displace the non-specific beads and capture maximum number of specific beads as compared to other frequencies (Fig. S5, videos 2-4, ESIt). At frequencies higher than $100 \mathrm{~Hz}$ at $V_{\mathrm{pp}}=4 \mathrm{~V}$, the shear forces become weaker and a large number of non-specific beads were attached to the electrode surface (Fig. S5, ESIt). On the other hand, field strengths under $f=100 \mathrm{~Hz}$ and $V_{\mathrm{pp}}=<4 \mathrm{~V}$, resulted in the beads moving more slowly and taking longer time to interact with the electrode surface (data not shown). However, at $f=100 \mathrm{~Hz}$

(A)

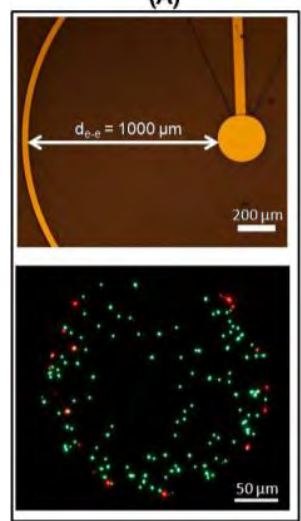

Fig. 2 (A) Brightfield microscopic image of an asymmetric electrode pair. The edge to edge distance between inner and outer electrode $\left(d_{e-e}\right)=1000 \mu \mathrm{m}$. The bottom fluorescence micrograph shows the capture of $50 \%$ specific beads in the presence of $50 \%$ non-specific beads at the field strength of $f=100 \mathrm{~Hz}$ and $V_{\mathrm{pp}}=$ 4V. (B) Shows the snapshots from real time video (Fig. S2, video 1, ESI+) showing the capture of streptavidin bead (green) and physical displacement of - $\mathrm{COOH}$ labelled bead (red) at the field strength of $f=100 \mathrm{~Hz}$ and $V_{\mathrm{pp}}=4 \mathrm{~V}$. The green colour bead attached to the electrode and red colour bead was displaced from the electrode surface due to the surface shear forces. 
and $V_{p p}=5 \mathrm{~V}$, the evolution of hydrogen gas due to electrolysis damaged the electrodes within one min of ac-EHD process (data not shown). Therefore, the field strength of $f=100 \mathrm{~Hz}$ and $V_{\mathrm{pp}}=4 \mathrm{~V}$ was selected for all ac- EHD experiments.

The performance of devices with different electrode designs was tested for the capture of specific beads in the presence of nonspecific beads under the optimal ac-EHD field $\left(f=100 \mathrm{~Hz}\right.$ and $V_{\mathrm{pp}}=$ $4 \mathrm{~V})$. Fig. S6 shows that the electrode with de-e of $1000 \mu \mathrm{m}$ captured maximum number of specific beads compared to the electrodes with $\mathrm{d}_{\mathrm{e}-\mathrm{e}}$ of 200 and $50 \mu \mathrm{m}$ (Fig. S6, videos 2, 5 and 6, ESIt). These data clearly indicate that the distance between the two electrodes has pronounced effects both on facilitating the binding of specific species and enhancing the removal of non-specific binding events. Since the asymmetric pair of electrodes with the $d_{\text {e-e }}$ of $1000 \mu \mathrm{m}$ gives maximum effective collisions, it was selected for further experiments. In order to assess the capture efficiency of the electrodes, five different mixtures of the specific and non-specific beads $(100 \%$ non-specific, $75 \%$ non-specific $+25 \%$ specific, $50 \%$ nonspecific $+50 \%$ specific, $25 \%$ non-specific $+75 \%$ specific and $100 \%$ specific) were analyzed using the optimal experimental parameters (Fig. 3). In each mixture, the total number of the specific and nonspecific beads were similar to that of either $100 \%$ specific or $100 \%$ non-specific beads (Fig. 3, Fig. S7, videos 2, 7-10, ESI†). The left panel
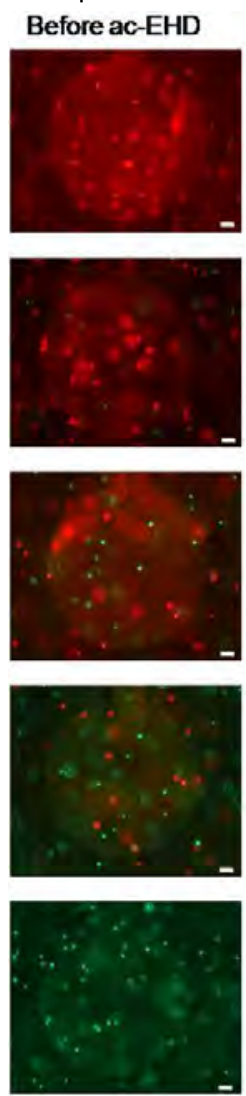
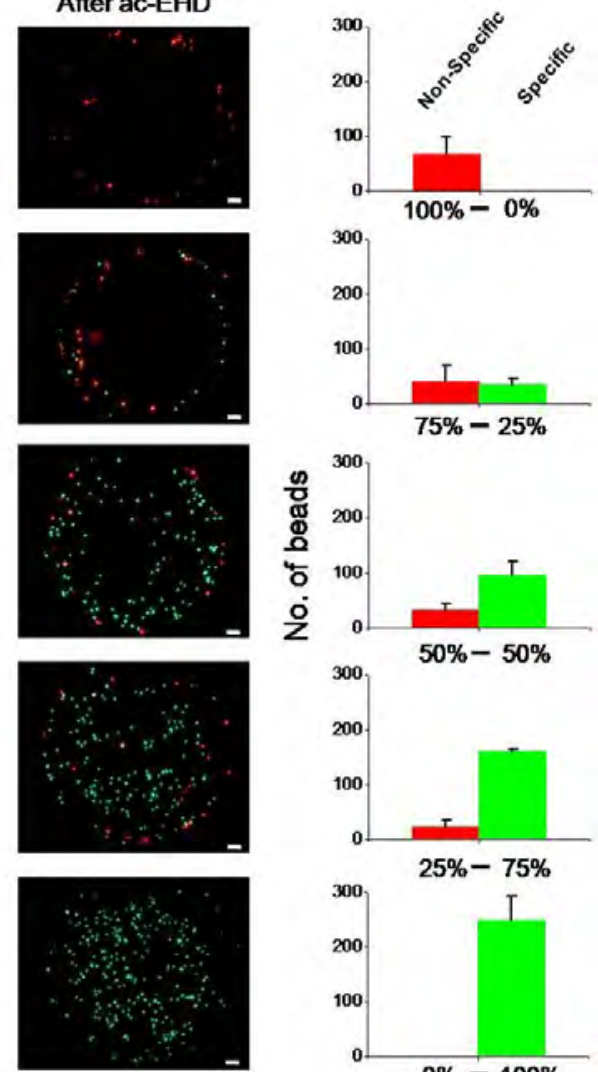

Fig. 3 Electrohydrodynamic surface shear force effect showing the decrease in non-specific adsorption and increase in specific capture in the mixtures of streptavidin and - $\mathrm{COOH}$ labelled beads. The left panel shows the snapshot of the beads just before the start of ac-EHD. The middle panel shows the bead capture after ac-EHD. The right panel shows the average and standard deviation of three experiments in each case. The ac field strength parameters were $f=100 \mathrm{~Hz}$ and $V_{\mathrm{pp}}=4 \mathrm{~V}$. The central electrode diameter $=250 \mu \mathrm{m}$ and $\mathrm{d}_{\mathrm{e}-\mathrm{e}}=1000 \mu \mathrm{m}$. in Fig. 3 shows the snapshots from the analysis chamber taken just before applying the ac-EHD. Clearly, the beads were floating in the PBS buffer under the Brownian motion just before ac-EHD. The middle panel shows the fluorescence micrographs obtained after performing ac-EHD induced capture process. To quantify an accurate number of the captured (specific or non-specific) beads, the devices were washed three times with PBS before taking these images. The right panel in Fig. 3 shows the quantitative representation of the specific and non-specific beads attached on the electrode surface after the application of ac-EHD. It can be seen that capture efficiency of $100 \%$ specific beads is approximately three-fold higher compared to that of the $100 \%$ non-specific beads (a similar number of beads were used in both experiments). In case of 50/50\% mixture of specific and non-specific beads, a similar level of enhancement in capture efficiency of specific beads was found. Capture efficiency of the $25 \%$ specific beads in presence of the $75 \%$ non-specific beads was over $2-$ fold higher compare to that of the $25 \%$ non-specific beads in presence of the $75 \%$ specific one. These data clearly indicate that acEHD effect was able to specifically capture target beads in the presence of the 3-fold higher non-specific beads.

In conclusion, we have demonstrated the real-time visualization of ac-EHD induced tuneable surface shear force and fluid mixing to physically displace non-specific beads from solid surface. Since the magnitude of the surface shear forces can be tuned externally (via changing the ac electric field), it can be readily adjustable to preferentially select strongly bound target beads over relatively weakly bound non-target beads. We believe that this phenomenon could have wide applications as an innovative approach to detect other targets (protein, cell, bacteria, etc) in biological samples.

This work was supported by the ARC DECRA (DE120102503) awarded for MJAS. We also acknowledge funding received by our laboratory from the National Breast Cancer Foundation of Australia (CG-08-07 and CG-12-07) to MT. These grants have significantly contributed to the environment to stimulate the research described here. The fabrication work was performed at Queensland node of the Australian National Fabrication Facility (Q-ANFF).

\section{Notes and references}

1. R. J. Hunter, Foundations of colloidal science, second edition edn., Oxford University Press Inc., New York, 1987.

2. Y. G. Li, Y. T. H. Cu and D. Luo, Nat Biotechnol, 2005, 23, 885-889.

3. S. Rauf, A. Glidle and J. M. Cooper, Chem Commun, 2010, 46, 2814-2816.

4. E. I. Galanzha, E. V. Shashkov, T. Kelly, J. W. Kim, L. L. Yang and V. P. Zharov, Nat Nanotechnol, 2009, 4, 855-860.

5. Y. L. Wang, K. Lee and J. Irudayaraj, Chem Commun, 2010, 46, 613-615.

6. S. S. Kumar, K. Kwak and D. Lee, Anal Chem, 2011, 83, 3244-3247.

7. L. Olofsson, T. Rindzevicius, I. Pfeiffer, M. Kall and F. Hook, Langmuir, 2003, 19, 10414-10419.

8. X. Yao, X. Li, et al., Anal Biochem, 2006, 354, 220-228.

9. M. Trau, D. A. Saville and I. A. Aksay, Science, 1996, 272, 706-709.

10. B. Bharti, G. H. Findenegg and O. D. Velev, Sci Rep-Uk, 2012, 2.

11. F. D. Ma, D. T. Wu and N. Wu, J Am Chem Soc, 2013, 135, 78397842.

12. A. Ramos, A. Gonzalez, A. Castellanos, N. G. Green and H. Morgan, Phys Rev E, 2003, 67. 


\section{ChemComm}

\section{Accepted Manuscript}
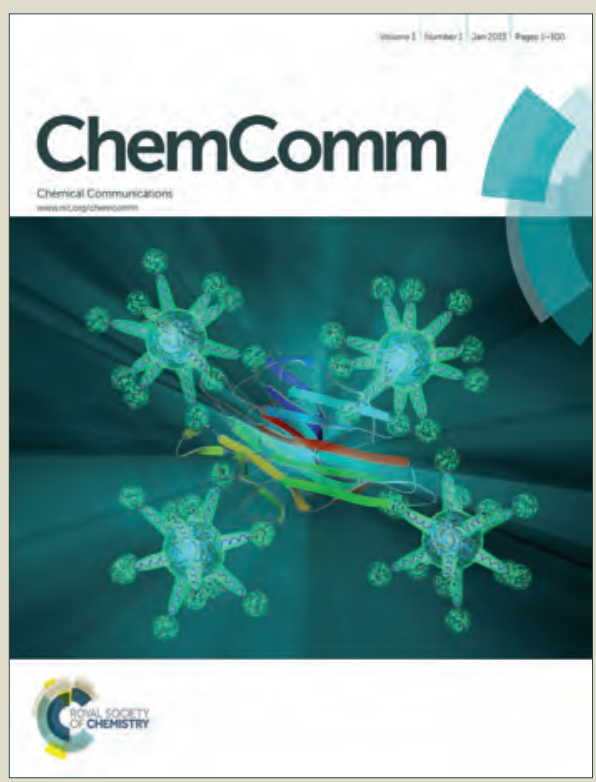

This is an Accepted Manuscript, which has been through the Royal Society of Chemistry peer review process and has been accepted for publication.

Accepted Manuscripts are published online shortly after acceptance, before technical editing, formatting and proof reading. Using this free service, authors can make their results available to the community, in citable form, before we publish the edited article. We will replace this Accepted Manuscript with the edited and formatted Advance Article as soon as it is available.

You can find more information about Accepted Manuscripts in the Information for Authors.

Please note that technical editing may introduce minor changes to the text and/or graphics, which may alter content. The journal's standard Terms \& Conditions and the Ethical guidelines still apply. In no event shall the Royal Society of Chemistry be held responsible for any errors or omissions in this Accepted Manuscript or any consequences arising from the use of any information it contains. 